\title{
Die Bestimmung von Blei, Nickel und Zink durch Fällung als Oxalate und deren Titration mit Kaliumpermanganat.
} Von

\author{
H. L. WARD. ${ }^{1}$
}

\section{Bestimmung von Blei.}

Bei seinen Untersuchungen über die Anwendung von Oxalaten in der Analyse fällte Rers ${ }^{2}$ Blei als Oxalat durch Zusatz von Ammoniumoxalat zu einer neutralen Lösung des Bleisalzes und Zersetzung des entstehenden löslichen Doppeloxalats mit einem groBen Überschub von Essigsäure. Das unlösliche Bleioxalat filtrierte er $a b$, verglühte es und brachte das Blei als Oxyd zur Wägung. Man konnte annehmen, daB das unter diesen Verhältnissen gefällte Bleioxalat eine derartige Zusammensetzung besäße, daß die Titration mit Permanganat eine genaue Bestimmung der vorhandenen Bleimenge lieferte.

Bei den in Tabeile 1 zusammengestellten Versuchen fällte man das Oxalat durch Zusatz von festem Ammoniumoxalat zur siedenden Bleinitratlösung, die bestimmte Mengen Essigsäure enthielt. Den Niederschlag sammelte man auf Asbest im Filtertiegel und wusch ihn mit kleinen Mengen Wasser. Dann wurde die Oxalsäure mit warmer verdünnter Schwefelsäure freigemacht und mit Kaliumpermanganat titriert.

Man sieht aus dem ersten Teil der Tabelle, wo die Essigsäuremengen $1 / 4$ der Lösung nicht überstiegen, daß die Fällung unvollständig ist. Wenn jedoch die Hälfte des Volumens der Flüssigkeit aus Eisessig besteht, dann sind die Ergebnisse genau.

Eine Anzahl von Chemikern ${ }^{3}$ haben als Fällungsmittel für Blei Oxalsäure verwendet. Die Bestimmungen von Tabelle 2 wurden ausgeführt durch Zusatz kristallisierter Oxalsäure zu einer sieden-

1 Aus dem Amer. Journ. Sci. (Sill.) ins Deutsche übertragen von I. KoppeLBerlin.

${ }^{2}$ Ber, deutsch. chem. Ges. 13, 502.

${ }^{3}$ Böttger u. Pollatz, Chem. Abstr. 2, 645. - Mohr-Classen, Lehrb. d. chem.-analyt. Titriermethode, S. 228. - Low, Journ. Amer. Chem. Soc. 30, 587 
Tabelle 1.

Bestimmung von Blei durch Fällung mit Ammoniumoxalat in Gegenwart von starker Essigsäure.

\begin{tabular}{c|c|c|c|c|c}
\hline $\begin{array}{c}\text { Blei, angew. } \\
\text { als Nitrat } \\
\mathrm{g}\end{array}$ & $\begin{array}{c}\text { Vol. bei der } \\
\text { Fällung } \\
\text { cem }\end{array}$ & $\begin{array}{c}\text { Vorhandene } \\
\text { Essigsäure } \\
\text { ccm }\end{array}$ & $\begin{array}{c}\text { Ammon- } \\
\text { oxalat } \\
\mathrm{g}\end{array}$ & $\begin{array}{c}\text { Blei, } \\
\text { gef. } \\
\mathrm{g}\end{array}$ & $\begin{array}{c}\text { Fehler } \\
\mathrm{g}\end{array}$ \\
\hline \hline 0.0050 & 100 & 25 & 3 & 0.0016 & -0.0036 \\
0.0250 & 100 & 25 & 3 & 0.0246 & -0.0004 \\
0.0500 & 100 & 5 & 3 & 0.0454 & -0.0046 \\
0.0500 & 100 & 10 & 3 & 0.0480 & -0.0020 \\
0.0500 & 100 & 25 & 3 & 0.0496 & -0.0004 \\
0.0500 & 200 & 50 & 7 & 0.0477 & -0.0023 \\
& & 50 & 4 & 0.0048 & -0.0002 \\
0.0050 & 100 & 50 & 4 & 0.0045 & -0.0005 \\
0.0050 & 100 & 50 & 4 & 00256 & +0.0006 \\
0.0250 & 100 & 50 & 4 & 0.0250 & \pm 0.0000 \\
0.0250 & 100 & 50 & 4 & 0.0505 & +0.0005 \\
0.0500 & 100 & 50 & 8 & 0.1002 & +0.0002 \\
0.1000 & 200 & 100 & & &
\end{tabular}

Tabelle 2.

Bestimmung von Blei als Oxalat durch Fällung mit Oxalsäure in Gegenwart starker Essigsäure.

\begin{tabular}{c|c|c|c|c|c|c}
\hline $\begin{array}{c}\text { Blei, angew. } \\
\text { als Nitrat } \\
\mathrm{g}\end{array}$ & $\begin{array}{c}\text { Vol. bei der } \\
\text { Fällung } \\
\text { ccm }\end{array}$ & $\begin{array}{c}\text { Essig- } \\
\text { säure } \\
\mathrm{ccm}\end{array}$ & $\begin{array}{c}\text { Oxal- } \\
\text { säure } \\
\mathrm{g}\end{array}$ & $\begin{array}{c}\text { Salz- } \\
\text { zusatz } \\
\mathrm{g}\end{array}$ & $\begin{array}{c}\text { Blei, } \\
\text { gef. } \\
\mathrm{g}\end{array}$ & $\begin{array}{c}\text { Fehler } \\
\mathrm{g}\end{array}$ \\
\hline \hline 0.0500 & 50 & - & 2 & - & 0.0491 & -0.0009 \\
0.0500 & 100 & - & 4 & - & 0.0488 & -0.0012 \\
0.1000 & 50 & - & 2 & - & 0.0994 & -0.0006 \\
0.1000 & 100 & - & 4 & - & 0.0990 & -0.0010 \\
0.0050 & 50 & 25 & 1 & - & 0.0050 & +0.0000 \\
0.0250 & 50 & 25 & 1 & - & 0.0256 & +0.0006 \\
0.1000 & 100 & 50 & 2 & - & 0.1002 & +0.0002 \\
\end{tabular}

In Gegenwart von Kaliumacetat.

\begin{tabular}{l|r|r|r|r|r|r}
0.1000 & 50 & - & 2 & 0.2 & 0.0962 & -0.0038 \\
0.1000 & 100 & - & 4 & 0.2 & 0.0988 & -0.0012 \\
0.1000 & 100 & 50 & 2 & 0.2 & 0.0997 & -0.0003 \\
0.1000 & 100 & 50 & 2 & 0.2 & 0.1000 & \pm 0.0000
\end{tabular}

In Gegenwart von Ammoniumacetat.

\begin{tabular}{l|c|c|c|c|c|c}
0.0050 & 50 & 25 & 1 & 3.0 & 0.0040 & -0.0010 \\
0.0250 & 100 & 50 & 2 & 5.0 & 0.0227 & -0.0023 \\
0.1000 & 100 & 50 & 2 & 2.0 & 0.1000 & \pm 0.0000
\end{tabular}


den Lösung von Bleinitrat, Filtration, Waschen und Titration mit Permanganat wie vorher. Wenn keine Essigsäure vorhanden ist, so verläuft die Fällung nicht ganz vollständig, aber die Fehler sind viel kleiner als bei Anwendung von Ammoniumoxalat als Fällungsmittel, wie man aus einem Vergleich mit Tabelle 1 erkennt. Zusatz von Essigsäure in gleichem Volumen zur Lösung sichert vollständige Fällung, sogar in Gegenwart beträchtlicher Mengen von Ammoniumoder Kaliumsalzen, vorausgesetzt, daB die vorhandene Menge Bleisalz nicht zu gering ist.

\section{Bestimmung von Nickel.}

CLASSEN $^{1}$ hat gezeigt, daß Nickel vollständig gefällt werden kann durch Behandlung des löslichen Nickel-Kaliumoxalats mit einer großen Menge Essigsäure. Das unter diesen Umständen gebildete Oxalat kann zu Oxyd verglüht und als solches gewogen werden. Es schien wünschenswert zu bestimmen, ob dies Oxalat eine derartig konstante Zusammensetzung hätte, daß man das Nickel durch Titration der Oxalsäure mit Kaliumpermanganat bestimmen könnte. Zuerst schlug man den von CLASSEN angegebenen Weg ein. $\mathrm{Zu}$ einer Lösung mit einer bekannten Menge Nickelsulfat setzte man einen beträchtlichen Überschuß von Kaliumoxalat und erhitzte die Flüssigkeit zum Sieden, worauf das zuerst gebildete Oxalat als NickelKaliumoxalat in Lösung ging. Dann setzte man 2 Volumina Essigsäure hinzu, um das Nickeloxalat zu fällen. Es erwies sich als notwendig, wenn man einen filtrierbaren Niederschlag erhalten wollte, die Essigsäure heiß zu machen, und langsam, unter fortwährendem Rühren in die siedende Lösung des Doppelsalzes einlaufen zu lassen. Hierbei bildete sich ein flockiger Niederschlag, der in ein feines Pulver überging, wenn er einige Zeit bei $60-70^{\circ}$ stand. Durch Filtration und Titration mit Permanganat fand man, daB das unter diesen Verhältnissen erhaltene Oxalat Neigung zeigt, etwas von dem Fällungsmittel einzuschließen, wodurch zu hohe Resultate bei der Titration erhalten wurden. Auch beim Glühen erhielt man große positive Fehler; wenn man jedoch das Oxyd auswusch, um das gebildete Kaliumkarbonat zu entfernen, so konnte man eine sehr gute Bestimmung des vorhandenen Nickels ausführen.

Es schien möglich, daB die Benutzung von Oxalsäure als Fällungsmittel diesen durch Inklusion bedingten Fehler ausschalten könnte.

1 Zeitschr. analyt. Chem. 16, 470. 
Aber man fand, daß bei Zusatz von Oxalsäure zur siedenden Lösung eines Nickelsalzes, die das gleiche Volumen Essigsäure enthielt, das Oxalat sich schnell in außerordentlich fein verteiltem Zustand abschied. Der erste Versuch von Tabelle 3 wurde in dieser Weise ausgeführt, aber in den meisten Fällen erwies sich die Filtration als unmöglich. Wenn jedoch die Fällung in wässeriger Lösung erfolgte und später Essigsäure zugesetzt wurde, um die letzten Spuren Nickel unlöslich zu machen, so fiel das Oxalat langsamer und in einer für die Filtration geeigneten Form aus.

Das zur Analyse dienende Nickel löste man in Wasser, und den Gehalt dieser Lösung bestimmte man durch Fällung als Nickelihydroxyd und Verglühen zu Oxyd, sowie durch Abscheidung als Metall an der rotierenden Kathode. Zu einem bestimmten Teil dieser Lösung, die auf das erforderliche Volumen verdünnt und zum Sieden erhitzt war, fügte man einen Überschuß kristallisierter Oxalsäure zu. Nach dem Abkühlen ließ man Essigsäure einfließen, und den Niederschlag ließ man über Nacht absitzen. Die kleineren Nickelmengen fielen nicht aus der wässerigen Lösung heraus, und selbst nach Zusatz von Essigsäure war es notwendig zu erhitzen, um die Fällung einzuleiten. Das Nickeloxalat filtrierte man über Asbest in einem Filtertiegel ab und wusch es mit kleinen Mengen Wasser. Den Tiegel brachte man in einen Becher mit $25 \mathrm{ccm}$ rerdünnter Schwefelsäure (1:4) und ließ die Auslösung des Oxalats in der Hitze erfolgen. Dann verdünnte man die Lösung auf etwa $200 \mathrm{ccm}$ und setzte solange Kobaltsulfat $\mathrm{zu}$, bis eine schwach rötliche Färbung auftrat. Dies von GibBs vorgeschlagene Verfahren war notwendig, um einen scharfen Endpunkt zu erhalten, da die

Tabelle 3.

Bestimmung von Nickel durch Fällung mit Oxalsäure und Bebandlung mit Essigsäure.

\begin{tabular}{c|c|c|c|c|c}
\hline $\begin{array}{c}\text { Nickel, angew. } \\
\text { als Sulfat } \\
\mathrm{g}\end{array}$ & $\begin{array}{c}\text { Vol. d. Lösg. } \\
\text { bei d. Fällung } \\
\text { cern }\end{array}$ & $\begin{array}{c}\text { Oxal } \\
\text { säure } \\
\mathrm{g}\end{array}$ & $\begin{array}{c}\text { Essigsäure- } \\
\text { zusatz } \\
\text { cem }\end{array}$ & $\begin{array}{c}\text { Nickel, } \\
\text { gef. } \\
\mathrm{g}\end{array}$ & $\begin{array}{c}\text { Fehler } \\
\mathrm{g}\end{array}$ \\
\hline \hline 0.0503 & 100 & 2 & 50 & 0.0502 & -0.0001 \\
0.0050 & 100 & 2 & 100 & 0.0054 & +0.0004 \\
0.0251 & 100 & 2 & 100 & 0.0259 & +0.0007 \\
0.0503 & 50 & 2 & 100 & 0.0514 & +0.0011 \\
0.0503 & 100 & 2 & 100 & 0.0502 & -0.0001 \\
0.1257 & 100 & 2 & 100 & 0.1271 & +0.0014 \\
& & & & &
\end{tabular}


grüne Farbe des Nickels das komplementäre Rot des Permanganats verdeckte. Der Becher wurde damn zum Sieden erhitzt und die Titration in der üblichen Weise ausgeführt. Die Ergebnisse sind in Tabelle 3 zusammengestellt. Die positiven Fehler können zwei Ursachen zugeschrieben werden: Einschluß des Fällungsmittels und Unsicherheit des Endpunktes bei der Titration gefärbter Lösungen.

\section{Bestimmung von Zink als 0xalat.}

Es erwies sich als möglich, das Zink volumetrisch als Oxalat nach demselbeu Verfahren zu bestimmen, das soeben für Nickel beschrieben ist. Da in diesem Fall in der Lösung wäbrend der Titration keine gefärbten Salze vorhanden sind, so erhielt man einen besseren Endpunkt und die Ergebnisse sind viel genauer, wie man aus Tabelle 4 sieht.

Tabelle 4.

Bestimmung von Zink durch Fällung mit Oxalsäure und Behandlung mit Essigsäure.

\begin{tabular}{c|c|c|c|c|c}
\hline $\begin{array}{c}\text { Zink, angew. } \\
\text { als Acetat } \\
\mathrm{g}\end{array}$ & $\begin{array}{c}\text { Vol. bei } \\
\text { der Fällung } \\
\text { ccm }\end{array}$ & $\begin{array}{c}\text { Oxal- } \\
\text { säure } \\
\mathrm{g}\end{array}$ & $\begin{array}{c}\text { Essigsäure } \\
\text { ccm }\end{array}$ & $\begin{array}{c}\text { Zink, } \\
\text { gef. } \\
\mathrm{g}\end{array}$ & $\begin{array}{c}\text { Fehler } \\
\mathrm{g}\end{array}$ \\
\hline \hline 0.0055 & 100 & 2 & 100 & 0.0056 & +0.0001 \\
0.0274 & 100 & 2 & 100 & 0.0276 & +0.0002 \\
0.0548 & 50 & 2 & 50 & 0.0553 & +0.0005 \\
0.0548 & 100 & 2 & 100 & 0.0550 & +0.0002 \\
0.1370 & 100 & 2 & 100 & 0.1372 & +0.0002
\end{tabular}

Das nach dem Verfahren von CLassen erhaltene Zink war mit viel Kaliumoxalat verunreinigt und konnte deswegen nicht zur Bestimmung der vorhandenen Zinkmenge benutzt werden. Als man den Niederschlag verglühte und das Oxyd auswusch, erkannte man, daß alles Zink gefällt war.

\section{Zusammenfassung.}

Es sind Versuche beschrieben worden, welche zeigen, daB Blei bestimmt werden kann durch Fällung mit Ammoniumoxalat oder Oxalsäure in Gegenwart großer Mengen Essigsäure und Titration des gebildeten Oxalats mit Permanganat.

Z. anorg. Chem. Bd. 77. 
Nickel wurde bestimmt durch Fällung mit Oxalsäure in wässeriger Lösung, Zusatz von Essigsäure zur Abscheidung des in Lösung verbleibenden Nickels und Titration mit Permanganat. Bei diesem Verfahren können Fehler auftreten durch Inklusion des Fällungsmittels oder einen mangelhaften Eindpunkt.

Zink kann sehr genau nach der beim Nickel angewendeten Methode bestimmt werden.

New Haren, U.S. A., The Kent Chemical Laboratory of Yale University.

Bei der Redaktion eingegangen am 6. Juni 1912. 